\title{
THE SECTORIAL PROJECTION DEFINED FROM LOGARITHMS
}

\author{
GERD GRUBB
}

\begin{abstract}
For a classical elliptic pseudodifferential operator $P$ of order $m>0$ on a closed manifold $X$, such that the eigenvalues of the principal symbol $p_{m}(x, \xi)$ have arguments in $] \theta, \varphi[$ and $] \varphi, \theta+2 \pi[$ $(\theta<\varphi<\theta+2 \pi)$, the sectorial projection $\Pi_{\theta, \varphi}(P)$ is defined essentially as the integral of the resolvent along $e^{i \varphi} \overline{\mathbf{R}}_{+} \cup e^{i \theta} \overline{\mathbf{R}}_{+}$. In a recent paper, Booss-Bavnbek, Chen, Lesch and Zhu have pointed out that there is a flaw in several published proofs that $\Pi_{\theta, \varphi}(P)$ is a $\psi$ do of order 0 ; namely that $p_{m}(x, \xi)$ cannot in general be modified to allow integration of $\left(p_{m}(x, \xi)-\lambda\right)^{-1}$ along $e^{i \varphi} \overline{\mathbf{R}}_{+} \cup e^{i \theta} \overline{\mathbf{R}}_{+}$simultaneously for all $\xi$. We show that the structure of $\Pi_{\theta, \varphi}(P)$ as a $\psi$ do of order 0 can be deduced from the formula $\Pi_{\theta, \varphi}(P)=\frac{i}{2 \pi}\left(\log _{\theta} P-\log _{\varphi} P\right)$ proved in an earlier work (coauthored with Gaarde). In the analysis of $\log _{\theta} P$ one need only modify $p_{m}(x, \xi)$ in a neighborhood of $e^{i \theta} \overline{\mathrm{R}}_{+}$; this is known to be possible from Seeley's 1967 work on complex powers.
\end{abstract}

\section{Functions of an elliptic operator}

Let $P$ be a classical elliptic pseudodifferential operator $(\psi \mathrm{do})$ of order $m>$ 0 acting in an $N$-dimensional hermitian vector bundle $E$ over a closed $n$ dimensional $C^{\infty}$ manifold $X$.

The construction of functions of $P$ was initiated by Seeley, who in [14] constructed and analysed the complex powers $P^{s}$ and showed that they are likewise classical $\psi$ do's, under the assumption that $P$ has one ray of minimal growth $\left\{\lambda=r e^{i \theta} \mid r \in \mathrm{R}_{+}\right\}$, where $(P-\lambda)^{-1}$ is well-defined and is $O\left(\lambda^{-1}\right)$ for $\lambda \rightarrow \infty$. They are useful in index theory for elliptic operators, and its generalizations, see also Atiyah and Bott [1]. Greiner [7] defined the heat operator $e^{-t P}$, when all rays with argument in $] \pi / 2-\delta, 3 \pi / 2+\delta[$ are rays of minimal growth (for some $\delta>0$ ); it is likewise used in index theory. The sectorial projection $\Pi_{\theta, \varphi}(P)$ is defined when $P$ has two rays of minimal growth $e^{i \theta} \mathbf{R}_{+}$and $e^{i \varphi} \mathbf{R}_{+}(\theta<\varphi<\theta+2 \pi)$, as a projection whose range includes the generalized eigenspaces for eigenvalues with argument in $] \theta, \varphi[$, and whose nullspace contains the generalized eigenspaces for eigenvalues with argument in ] $\varphi, \theta+2 \pi$ [. Burak [5] studied it for $P$ equal to a realization $A_{B}$ of an elliptic differential operator $A$ with a boundary condition $B u=0$. A special case, the

Received 24 February 2011, in final form 28 July 2011. 
positive eigenprojection $\Pi_{+}(P)$ for a selfadjoint differential or pseudodifferential operator, came into focus with the works of Atiyah, Patodi and Singer [2], [3] on index formulas for Dirac operators with boundary conditions; here $\Pi_{+}(P)$ equals $(P+|P|)(2|P|)^{-1}$ (defined to be zero on the nullspace of $P$ ), and is a $\psi$ do of order 0 since $|P|=\left(P^{2}\right)^{\frac{1}{2}}$ is classical elliptic of order $m$ by [14]. Wodzicki [15], [16], [17], in his studies of the spectral asymmetry, considered $\Pi_{+}(P)$, as well as more general sectorial projections in cases where $P$ has two rays of minimal growth. The logarithm $\log _{\theta} P$ is defined when $e^{i \theta} \mathrm{R}_{+}$ is a ray of minimal growth, and arises e.g. as the derivative of $P^{s}$ at $s=0$; it was analysed in detail by Okikiolu [10], [11] in connection with determinant formulas.

The sectorial projection can be defined on smooth functions by the formula

$$
\Pi_{\theta, \varphi}(P)=\frac{i}{2 \pi} \int_{\Gamma_{\theta, \varphi}} \lambda^{-1} P(P-\lambda)^{-1} d \lambda,
$$

where the integration goes along the sectorial contour

$$
\begin{aligned}
\Gamma_{\theta, \varphi}=\left\{r e^{i \varphi} \mid \infty>r\right. & \left.>r_{0}\right\} \\
& \cup\left\{r_{0} e^{i \omega} \mid \varphi \geq \omega \geq \theta\right\} \cup\left\{r e^{i \theta} \mid r_{0}<r<\infty\right\} ;
\end{aligned}
$$

here $r_{0}$ is taken so small that 0 is the only possible eigenvalue in $\left\{|\lambda| \leq r_{0}\right\}$.

Detailed studies of $\Pi_{\theta, \varphi}(P)$ were also made by Wojciechowski [18] for applications to the spectral flow for first-order operators, by NazaikinskiiSternin-Shatalov-Schulze [9] for manifolds with singularities, and by Ponge [12] who wanted to give a simplified proof of the results of Wodzicki. A recent paper of Booss-Bavnbek, Chen, Lesch and Zhu [4] gives an interesting observation, namely that there is a flaw in the arguments of the latter three papers, where the construction of $\Pi_{\theta, \varphi}(P)$ is based on an application of (1) to the terms in the symbol of $(P-\lambda)^{-1}$ : When the principal symbol $p_{m}(x, \xi)$ has eigenvalues both with arguments in $] \theta, \varphi[$ and in $] \varphi, \theta+2 \pi[$, one cannot obtain that $\left(p_{m}(x, \xi)-\lambda\right)^{-1}$ is nonsingular along the curve $\Gamma_{\theta, \varphi}$ for all $\xi \in \mathrm{R}^{n}$; there is a topological obstruction (see the detailed explanation in [4]). Therefore a modified proof is needed.

The reader is referred to the paper of Booss-Bavnbek et al. for their strategy to circumvent the mentioned difficulty. They show that $\Pi_{\theta, \varphi}(P)$ is $H^{s}$-bounded, when $P$ has a homogeneous principal symbol and a lower-order part in $S_{1,0}^{m-1}$. They use their estimates to show that the norm of $\Pi_{\theta, \varphi}(P)$ in $H^{s}$-spaces depends continuously on $P$ in a certain symbol/operator topology coarser than the full symbol topology.

We shall here show, when $P$ is classical, that a very easy proof of the fact that $\Pi_{\theta, \varphi}(P)$ is a classical $\psi$ do of order 0 (in particular $H^{s}$-bounded) comes 
from the relation between the sectorial projection and logarithms of $P$, as worked out in detail in Gaarde-Grubb [6].

\section{Preliminaries on the logarithm of $P$}

The present author's interest in the logarithm stems from reading the paper of Scott [13], where it was shown that $C_{0}(P)=-\frac{1}{m} \operatorname{res}(\log P)$; here $C_{0}(P)=$ $\zeta(P, 0)+\operatorname{dim} \operatorname{ker} P$, where $\zeta(P, s)$ is the meromorphic extension of $\operatorname{Tr} P^{-s}$, and the residue of $\log P$ is as defined in Okikiolu [11]. Since $C_{0}(P)$ is also equal to the coefficient of $-\lambda^{-1}$ in the expansion of the resolvent trace $\operatorname{Tr}(P-\lambda)^{-1}$ (take $m>n$ for simplicity in this motivating explanation), this coefficient is related in the same way to $\operatorname{res}(\log P)$. We wanted to give a direct proof of the last fact without having to calculate complex powers - for the sake of a generalization to boundary value problems where complex powers are difficult to use. The outcome is explained in [8], where the point of departure is a simple key lemma (Lemma 1.2) that shows how the logarithm comes into the resolvent trace calculations. This was used to show Scott's formula directly from resolvent trace expansions, and the method was generalized to get similar results for manifolds with boundary. Related observations were used to deduce the results in [6] that we appeal to in Section 3 below.

Assume in this section that $P$ is elliptic of order $m \in \mathbf{R}_{+}$, having a ray of minimal growth $e^{i \theta} \mathbf{R}_{+}$for some $\theta \in[0,2 \pi$ [. This means that the principal symbol $p_{m}(x, \xi)$, homogeneous of degree $m$ in $\xi$ for $|\xi| \geq 1$ and smooth in $(x, \xi)$, has no eigenvalues on $e^{i \theta} \mathrm{R}_{+}$when $|\xi| \geq 1$. Then $(P-\lambda)^{-1}$ exists and is $O\left(\lambda^{-1}\right)$ for large $\lambda$ on the ray, and since the hypotheses are valid also for rays with argument close to $\theta$, one can assume that the ray is free of eigenvalues of $P$.

The principal symbol of the resolvent is $q_{-m}(x, \xi, \lambda)=\left(p_{m}(x, \xi)-\lambda\right)^{-1}$ for $|\xi| \geq 1$, assumed to be extended in a smooth way for $|\xi| \leq 1$. The smoothing can be done for each $\lambda$ e.g. by multiplication of $\left(p_{m}^{h}(x, \xi)-\lambda\right)^{-1}$ (where $p_{m}^{h}(x, \xi)$ denotes the strictly homogeneous symbol) by an excision function $\zeta(\xi)$ (a nonnegative $C^{\infty}$ function that equals 1 for $|\xi| \geq 1,0$ near $\xi=0$ ). In some cases it suffices to modify $p_{m}(x, \xi)$ itself for small $\xi$.

The lower order terms $q_{-m-j}$ in the symbol $q(x, \xi, \lambda)$ of $(P-\lambda)^{-1}$ are defined in local coordinates by recursive formulas known from [14]; they are finite sums of terms with the structure

$$
r(x, \xi, \lambda)=b_{1} q_{-m}^{\nu_{1}} b_{2} q_{-m}^{\nu_{2}} \ldots b_{M} q_{-m}^{\nu_{M}} b_{M+1},
$$

where the $b_{k}$ are homogeneous $\psi$ do symbols independent of $\lambda$, the $v_{k}$ are positive integers with sum $\geq 2$. $(P-\lambda)^{-1}$ has the full symbol $q(x, \xi, \lambda) \sim$ 
$\sum_{j \geq 0} q_{-m-j}(x, \xi, \lambda)$. The terms $q_{-m-j}$ are quasi-homogeneous (homogeneous of degree $-m-j$ in $\left(\xi,|\lambda|^{\frac{1}{m}}\right)$ on each ray).

Now $\log _{\theta} P$ can be defined on smooth functions by

$$
\log _{\theta} P=\lim _{s \searrow 0} \frac{i}{2 \pi} \int_{\mathscr{C}} \lambda_{\theta}^{-s} \log _{\theta} \lambda(P-\lambda)^{-1} d \lambda
$$

Here $\lambda_{\theta}^{-s}$ and $\log _{\theta} \lambda$ are taken with branch cut $e^{i \theta} \mathrm{R}_{+}$, and $\mathscr{C}$ is a contour in $\mathrm{C} \backslash e^{i \theta} \overline{\mathrm{R}}_{+}$going around the nonzero spectrum of $P$ in the positive direction; for precision we can take a Laurent loop

$$
\begin{aligned}
\mathscr{C}_{\theta}=\left\{r e^{i \theta} \mid \infty>r>r_{0}\right\} \\
\cup\left\{r_{0} e^{i \omega} \mid \theta \geq \omega \geq \theta-2 \pi\right\} \cup\left\{r e^{i(\theta-2 \pi)} \mid r_{0}<r<\infty\right\},
\end{aligned}
$$

with $r_{0}$ so small that 0 is the only possible eigenvalue in $\left\{|\lambda| \leq r_{0}\right\}$. As shown in Okikiolu [10], the symbol of $\log _{\theta} P$ is calculated in local coordinates from the resolvent symbol $q(x, \xi, \lambda)$ by integration with $\log _{\theta} \lambda$ around the spectrum of the principal symbol $p_{m}$. The terms $q_{-m-j}$ contribute as follows:

$$
\begin{aligned}
& \frac{i}{2 \pi} \int_{\mathscr{C}_{\theta}(x, \xi)} \log _{\theta} \lambda q_{-m}(x, \xi, \lambda) d \lambda \\
& \quad=\frac{i}{2 \pi} \int_{\mathscr{C}_{\theta}(x, \xi)} \log _{\theta} \lambda\left(p_{m}(x, \xi)-\lambda\right)^{-1} d \lambda \\
& \quad=\log _{\theta} p_{m}(x, \xi)=\log _{\theta}\left([\xi]^{m}\right)+\log _{\theta}\left([\xi]^{-m} p_{m}(x, \xi)\right) \\
& \quad=m \log [\xi]+l_{\theta, 0}(x, \xi), \\
& \frac{i}{2 \pi} \int_{\mathscr{C}_{\theta}(x, \xi)} \log _{\theta} \lambda q_{-m-j}(x, \xi, \lambda) d \lambda=l_{\theta,-j}(x, \xi) \quad \text { for } \quad j>0,
\end{aligned}
$$

where $\mathscr{C}_{\theta}(x, \xi)$ is a closed curve in $\mathrm{C} \backslash e^{i \theta} \overline{\mathrm{R}}_{+}$around the spectrum of $p_{m}(x, \xi)$, and $[\xi]$ stands for a smooth positive function on $\mathrm{R}^{n}$ equal to $|\xi|$ for $|\xi| \geq 1$. It is a point here that the Laurent loop used for $\log _{\theta} P$ is replaced by a closed curve (by replacement of the rays outside a large $R$ by an arc with radius $R$ ) and $\lambda^{-s}$ is replaced by its limit 1 , since the spectrum is bounded at each $(x, \xi)$. If, more generally, $\theta=\theta_{0}+2 \pi k$ with $\theta_{0} \in\left[0,2 \pi\right.$ [ and $k$ integer, then $l_{\theta, 0}$ contains an additional constant $2 \pi i k$.

Each $l_{\theta,-j}$ is homogeneous in $\xi$ of degree $-j$ for $|\xi| \geq 1$; for $j=0$ it follows since $[\xi]^{-m} p_{m}(x, \xi)$ is so, and for $j \geq 1$ it is seen e.g. as follows 
(where we set $\lambda=t^{m} \varrho, t \geq 1$ ):

$$
\begin{aligned}
& l_{\theta,-j}(x, t \xi) \\
& \quad= \frac{i}{2 \pi} \int_{\mathscr{C}_{\theta}(x, \xi)} \log _{\theta} \lambda q_{-m-j}(x, t \xi, \lambda) d \lambda \\
& \quad= \frac{i}{2 \pi} \int_{t^{-m} \mathscr{C}_{\theta}(x, \xi)}\left(\log _{\theta} \varrho+m \log t\right) t^{-m-j} q_{-m-j}(x, \xi, \varrho) t^{m} d \varrho \\
& \quad=t^{-j} l_{\theta,-j}(x, \xi)+m t^{-j} \log t \frac{i}{2 \pi} \int_{t^{-m} \mathscr{C}_{\theta}(x, \xi)} q_{-m-j}(x, \xi, \varrho) d \varrho,
\end{aligned}
$$

where the last term is zero since $q_{-m-j}$ is $O\left(|\varrho|^{-2}\right)$ for $|\varrho| \rightarrow \infty$ when $j>0$.

In the proof that the full symbol of $\log _{\theta} P$ is

$$
m \log [\xi]+l_{\theta}(x, \xi), \quad l_{\theta}(x, \xi) \sim \sum_{j \geq 0} l_{\theta,-j}(x, \xi),
$$

one uses the observation by Seeley [14] that the symbol $p_{m}(x, \xi)$ can be modified smoothly near $\xi=0$ in such a way that $p_{m}(x, \xi)-\lambda$ is invertible for all $\lambda$ in a keyhole region $\{\lambda \in \mathrm{C} \mid \arg \lambda \in] \theta-\delta, \theta+\delta[$ or $|\lambda|<r\}$, all $(x, \xi)$, when $r$ and $\delta$ are sufficiently small positive numbers.

\section{The relation between the sectorial projection and logarithms}

For a general closed, densely defined operator $A$ in a Hilbert space $H$, with compact resolvent and two rays $e^{i \theta} \mathbf{R}_{+}$and $e^{i \varphi} \mathbf{R}_{+}$in the resolvent set, where $\left\|(A-\lambda)^{-1}\right\|$ is $O\left(\lambda^{-1}\right)$ for $\lambda \rightarrow \infty$ on the rays, one defines $\Pi_{\theta, \varphi}(A)$ on $D(A)$, to begin with, by

$$
\Pi_{\theta, \varphi}(A) x=\frac{i}{2 \pi} \int_{\Gamma_{\theta, \varphi}} \lambda^{-1} A(A-\lambda)^{-1} x d \lambda, \quad x \in D(A) ;
$$

here the integration goes along the sectorial contour (2). If the hereby defined operator $\Pi_{\theta, \varphi}(A)$ is bounded in $H$-norm, we extend it by continuity to $H$.

Similarly, if $A$ has compact resolvent and one ray $e^{i \theta} R_{+}$in the resolvent set, where $\left\|(A-\lambda)^{-1}\right\|$ is $O\left(\lambda^{-1}\right)$ for $\lambda \rightarrow \infty$ on the ray, one can define $\log _{\theta} A$ by the formula

(10) $\log _{\theta} A x=\lim _{s \searrow 0} \frac{i}{2 \pi} \int_{\mathscr{C}} \lambda_{\theta}^{-s} \log _{\theta} \lambda(A-\lambda)^{-1} x d \lambda, \quad x \in D(A)$.

The results in the following theorem were shown in [6] (Lemma 4.3 and Prop. 4.4). 
THEOREM 3.1. $1^{\circ}$ Let $f(\lambda)$ be a continuous (possibly vector-valued) function on the "punctuated double keyhole region"

$$
V_{r_{0}, \delta}=\left\{\lambda \in \mathrm{C}|| \lambda \mid<2 r_{0} \text { or }|\arg \lambda-\theta|<\delta \text { or }|\arg \lambda-\varphi|<\delta\right\} \backslash\{0\},
$$

such that $f(\lambda)$ is $O\left(\lambda^{-1-\varepsilon}\right)$ for $|\lambda| \rightarrow \infty$ in $V_{r_{0}, \delta}$. Then

$$
\int_{\mathscr{C}_{\theta}} \log _{\theta} \lambda f(\lambda) d \lambda-\int_{\mathscr{C}_{\varphi}} \log _{\varphi} \lambda f(\lambda) d \lambda=-2 \pi i \int_{\Gamma_{\theta, \varphi}} f(\lambda) d \lambda .
$$

$2^{\circ}$ For $x \in D(A)$,

$$
\log _{\theta} A x-\log _{\varphi} A x=\int_{\Gamma_{\theta, \varphi}} \lambda^{-1} A(A-\lambda)^{-1} x d \lambda=-2 \pi i \Pi_{\theta, \varphi}(A) x .
$$

When $\Pi_{\theta, \varphi}(A)$ is bounded, so is $\log _{\theta} A-\log _{\varphi} A$ (and vice versa), and then

$$
\Pi_{\theta, \varphi}(A)=\frac{i}{2 \pi}\left(\log _{\theta} A-\log _{\varphi} A\right) .
$$

We now assume that $P$ is elliptic of order $m \in \mathbf{R}_{+}$, having two rays of minimal growth $e^{i \theta} \mathbf{R}_{+}$and $e^{i \varphi} \mathbf{R}_{+}$(with $\theta \in[0,2 \pi[, \theta<\varphi<\varphi+2 \pi$ ). Then $(P-\lambda)^{-1}$ exists and is $O\left(\lambda^{-1}\right)$ for large $\lambda$ on the rays, and we can assume that the rays are free of eigenvalues of $P$. The considerations in Theorem 3.1 will be applied to $P$, entering as a closed unbounded operator in $H=L_{2}(X)$ with domain $D(P)=H^{m}(X)$.

Theorem 3.2. $\Pi_{\theta, \varphi}(P)$ equals $\frac{i}{2 \pi}\left(\log _{\theta} P-\log _{\varphi} P\right)$, and is a classical $\psi d o$ of order $\leq 0$. It has the symbol

$$
\pi_{\theta, \varphi}(x, \xi)=\frac{i}{2 \pi}\left(l_{\theta}(x, \xi)-l_{\varphi}(x, \xi)\right),
$$

in local coordinates.

Proof. As recalled in Section 2, $\log _{\theta} P$ and $\log _{\varphi} P$ are $\log$-polyhomogeneous $\psi$ do's with symbols as described in (8). Then $\log _{\theta} P-\log _{\varphi} P$ has the symbol

$$
l_{\theta}(x, \xi)-l_{\varphi}(x, \xi),
$$

where the log-terms $m \log [\xi]$ have cancelled out. Hence it is a classical $\psi$ do of order $\leq 0$; in particular it is bounded on $L_{2}(X)$. By Theorem $3.12^{\circ}, \Pi_{\theta, \varphi}(P)$ is then also bounded on $L_{2}(X)$; and it equals $\frac{i}{2 \pi}\left(\log _{\theta} P-\log _{\varphi} P\right)$ and is a 
classical $\psi$ do on $X$ of order $\leq 0$ whose symbol is found as the corresponding linear combination of the symbols of the logarithms, namely (15).

We can also show that the terms in the symbol of $\Pi_{\theta, \varphi}(P)$ have the expected form as integrals of terms in the resolvent symbol:

THEOREM 3.3. In local coordinates, the symbol

$$
\pi_{\theta, \varphi}(x, \xi) \sim \sum_{j \geq 0} \pi_{\theta, \varphi,-j}(x, \xi)
$$

of $\Pi_{\theta, \varphi}(P)$ satisfies, for each $x$, each $|\xi| \geq 1$ :

$$
\begin{aligned}
\pi_{\theta, \varphi,-j}(x, \xi) & =\frac{i}{2 \pi}\left(l_{\theta,-j}(x, \xi)-l_{\varphi,-j}(x, \xi)\right) \\
& =\frac{i}{2 \pi} \int_{\mathscr{C}_{\theta, \varphi}(x, \xi)} q_{-m-j}(x, \xi, \lambda) d \lambda,
\end{aligned}
$$

for all $j$. Here $\mathscr{C}_{\theta, \varphi}(x, \xi)$ is a closed curve in the open sector

$$
\Lambda_{\theta, \varphi}=\{\lambda \in \mathrm{C} \mid \theta<\arg \lambda<\varphi\}
$$

going in the positive direction around the spectrum of $p_{m}(x, \xi)$ lying in that sector.

Proof. The first equality in (17) follows immediately from (15). For the second equality we use (6). For $j=0$, we obtain the formula by applying Theorem $3.12^{\circ}$ to the bounded operator $p_{m}(x, \xi)$ in $\mathrm{C}^{N}$ :

$$
\begin{aligned}
l_{\theta, 0}-l_{\varphi, 0} & =\log _{\theta} p_{m}-\log _{\varphi} p_{m}=\int_{\Gamma_{\theta, \varphi}} \lambda^{-1} p_{m}\left(p_{m}-\lambda\right)^{-1} d \lambda \\
& =\int_{\mathscr{C}} \lambda^{-1} p_{m}\left(p_{m}-\lambda\right)^{-1} d \lambda=\int_{\mathscr{C}}\left(\lambda^{-1}+\left(p_{m}-\lambda\right)^{-1}\right) d \lambda \\
& =\int_{\mathscr{C}}\left(p_{m}-\lambda\right)^{-1} d \lambda,
\end{aligned}
$$

where the curve $\Gamma_{\theta, \varphi}$ could be replaced by a closed curve $\mathscr{C}=\mathscr{C}_{\theta, \varphi}(x, \xi)$ in $\Lambda_{\theta, \varphi}$, since the integrand was $O\left(\lambda^{-2}\right)$ and the spectrum of $p_{m}(x, \xi)$ in $\Lambda_{\theta, \varphi}$ is a finite set of points. For $j \geq 1$, we obtain the formula by application of Theorem $3.11^{\circ}$ with $f(\lambda)$ equal to the $j$ 'th term $q_{-m-j}(x, \xi, \lambda)$ in the resolvent symbol (recall the structure as a sum of terms (3)); it is $O\left(\lambda^{-2}\right)$, allowing reduction to a closed curve $\mathscr{C}$. 
In relation to the problem raised in [4], we note that the calculations in Theorem 3.3 take place at individual points $(x, \xi)$, where there is no problem with singularities on the curve $\Gamma_{\theta, \varphi}$. Calculations global in $\xi$ are only performed in the constructions of the logarithms, where the argument of Seeley [14] is valid.

In [6] we relied on the account of Ponge [12] referring to five works of Wodzicki from the 80's (two in Russian), for the knowledge that $\Pi_{\theta, \varphi}(P)$ is a zero-order $\psi$ do. Although Ponge's own formulation of a proof has the flaw pointed out in [4], we see no reason to doubt the original statement, which is further supported by the formula $P_{\theta}^{s}-P_{\varphi}^{s}=\left(1-e^{2 i \pi s}\right) \Pi_{\theta, \varphi}(P) P_{\theta}^{s}$ in [12] Sect. 4, ascribed to Wodzicki.

At any rate, it seems to be useful that the present paper gives an independent proof which avoids the mentioned pitfall, and is based directly on resolvents and logarithms.

The formula $\Pi_{\theta, \varphi}(P)=\frac{i}{2 \pi}\left(\log _{\theta} P-\log _{\varphi} P\right)$ allows a direct application of the procedures and results in Okikiolu [10], Sect. 4, to show that the norm in $H^{s}$-spaces depends continuously and even smoothly on the symbol of $P$, in dependence on a parameter $t$ in an open subset $T$ of $\mathrm{R}^{d}$. As mentioned earlier, [4] shows the continuity in terms of a certain symbol/operator topology on $P$.

\section{REFERENCES}

1. Atiyah, M. F., and Bott, R., A Lefschetz fixed point formula for elliptic complexes I, Ann. of Math. (2) 86 (1967), 374-407.

2. Atiyah, M. F., Patodi, V. K., and Singer, I. M., Spectral asymmetry and Riemannian geometry I, II, Math. Proc. Cambridge Philos. Soc. 77 (1975), 43-69 and 78 (1975), 405-432.

3. Atiyah, M. F., Patodi, V. K., and Singer, I. M., Spectral asymmetry and Riemannian geometry III, Math. Proc. Cambridge Philos. Soc. 79 (1976), 71-99.

4. Booss-Bavnbek, B., Chen, G., Lesch, M., and Zhu, C., Perturbation of sectorial projections of elliptic pseudo-differential operators, to appear in J. Pseudo-Differ. Oper. Appl.

5. Burak, T., On spectral projections of elliptic operators, Ann. Scuola Norm. Sup. Pisa (3) 24 (1970), 209-230.

6. Gaarde, A., and Grubb, G., Logarithms and sectorial projections for elliptic boundary problems, Math. Scand. 103 (2008), 243-277.

7. Greiner, P., An asymptotic expansion for the heat equation, Arch. Rational Mech. Anal. 41 (1971), 163-218.

8. Grubb, G., On the logarithm component in trace defect formulas, Comm. Partial Differential Equations 30 (2005), 1671-1716.

9. Nazaikinskii, V., Sternin, B., Shatalov, V., and Schulze, B.-W., Spectral boundary value problems and elliptic equations on manifolds with singularities, Diff. Equ. 34 (1998), 696-710.

10. Okikiolu, K., The Campbell-Hausdorff theorem for elliptic operators and a related trace formula, Duke Math. J. 79 (1995), 687-722.

11. Okikiolu, K., The multiplicative anomaly for determinants of elliptic operators, Duke Math. J. 79 (1995), 723-750. 
12. Ponge, R., Spectral asymmetry, zeta functions, and the noncommutative residue, Internat. J. Math. 17 (2006), 1065-1090.

13. Scott, S., The residue determinant, Comm. Partial Differential Equations 30 (2005), 483-507.

14. Seeley, R. T., Complex powers of an elliptic operator, pp. 288-307 in: Singular Integrals, Proc. Symp. Pure Math. X, Proc. Chicago 1966, Amer. Math. Soc., Providence, RI 1967.

15. Wodzicki, M., Spectral asymmetry and zeta functions, Invent. Math. 66 (1982), 115-135.

16. Wodzicki, M., Local invariants of spectral asymmetry, Invent. Math. 75 (1984), 143-178.

17. Wodzicki, M., Noncommutative residue I. Fundamentals, pp. 320-399 in: K-theory, Arithmetic and Geometry, Proc. Moscow 1984-1986, Lecture Notes in Math. 1289, Springer, Berlin 1987.

18. Wojciechowski, K., Spectral flow and the general linear conjugation problem, Simon Stevin 59 (1985), 59-91.

DEPARTMENT OF MATHEMATICAL SCIENCES

COPENHAGEN UNIVERSITY

UNIVERSITETSPARKEN 5

DK-2100 COPENHAGEN

DENMARK

E-mail: grubb@math.ku.dk 